\title{
Multiparametric quality by design-fuzzy model applied in the development of a biomedical measuring system
}

\author{
Daniel René Tasé Velázquez ${ }^{1, *}$, Elisabeth Costa Monteiro ${ }^{2}$, Daniel Ramos Louzada ${ }^{2}$, \\ and Carlos Roberto Hall Barbosa ${ }^{2}$ \\ ${ }^{1}$ Postgraduate Programme in Production Engineering, Methodist University of Piracicaba, Rod. Luís Ometto km 24, Santa \\ Bárbara d'Oeste, SP, Brazil \\ 2 Postgraduate Programme in Metrology, Pontifical Catholic University of Rio de Janeiro, Marquês de São Vicente 225, Rio de \\ Janeiro,RJ, Brazil
}

Received: 21 October 2020 / Accepted: 27 October 2020

\begin{abstract}
This work presents the adaptation of the Quality by Design (QbD) approach for application in the quality assurance of a biomedical measuring system under development. First attempts in applying QbD to biomedical technologies indicated a significantly higher number of parameters than its traditional application in the pharmaceutical industry. These preliminary studies did not fulfill the QbD stage of Design Space (DS) configuration for biomedical devices, an essential step to identifying the proper operating ranges of parameters and guaranteeing quality features. Therefore, it persisted the challenge of configuring DS for health devices, overcoming dependences in the interaction of multiple process parameters and critical attributes. The present work develops a hybrid QbD-Fuzzy approach for multiparametric DS configuration. The proposed method was applied in the development phase of a low-cost and high-sensitive magnetic measuring system for locating metallic foreign bodies in patients, employing sensors based on the Giant Magnetoimpedance effect. The results provided the acceptable operating ranges of the multiple process parameters to ensure the biomedical equipment's suitability. The proposed strategy contributes to the QbD implementation in biomedical technologies and, therefore, promotes the reliability of diagnostic and therapeutic results in the clinical environment.
\end{abstract}

Keywords: Metrology / quality by design / fuzzy inference / magnetic transducer / biomedical device

\section{Introduction}

Biomedical devices are essential elements in the health sector routine. Quality control in the production processes of these technologies, as well as characteristics and parameters associated with their development, are topics of great interest in this industry, which is growing fast, with a broad market. Thus, the use of tools and methodologies aimed at guaranteeing quality and safety from the first design stages of these devices becomes crucial [1-8].

To ensure safe and effective diagnoses and therapeutic approaches, without risk of adverse events and with full access for the worldwide community, in the development of new technologies for biomedical application should be considered characteristics that meet the so-called Biometrological precepts $[7,8]$. These principles include low cost

\footnotetext{
* Corresponding author: dtasev88@gmail.com
}

of manufacture and operation, low complexity, portability, noninvasiveness, and innocuousness, besides appropriate accuracy and precision, providing results with metrological traceability to SI units (International System of Units) $[7,8]$.

According to recommendations of the Food and Drug Administration (FDA) in the USA [9,10], the Quality by Design (QbD) methodology has been applied since 2005 to ensure higher quality in pharmaceutical manufacturing. Based on the guidelines for quality management in drug production, it was introduced by the International Conference on Harmonization of Technical Requirements for Registration of Pharmaceuticals for Human Use (ICH) [11-14]. QbD is characterized as an approach that provides a better understanding of critical manufacturing processes and their relationship to the final product's quality. For that reason, control strategies can be defined based on established limits during the development phase, to guarantee the fulfillment of predefined quality objectives of products to be manufactured. This strategy results in 
a flexible manufacturing process that allows its adaptation and continuous improvement.

The fundamental assumption underlying QbD is that product quality can only be ensured if critical sources of variability are understood and adequately mitigated or controlled [15]. Thus, the QbD tool can be implemented by identifying a series of interdependent parameters and attributes, which, when analyzed, generate knowledge for optimization and quality assurance of the product to be fabricated [16].

The implementation of $\mathrm{QbD}$ is intended to design, evaluate and control production through testing to assess the quality and performance of critical materials and process-related elements, ensuring the safety of the final product [17].

For applying the QbD approach, the first step involves defining the Target Product Profile (TPP). TPP describes aspects associated with the use, safety, efficacy, and functionality of the product to be developed. From these characteristics, the Critical Quality Attributes (CQAs) are determined, which consist of critical attributes that must be met to guarantee the desired quality of the product. Finally, the Critical Process Parameters (CPPs) are established, which may be characterized by operating ranges, setting limits to their variations throughout the process, which may affect the quality of the final product.

Once TPPs, CQAs, and CPPs are defined, the Design Space (DS) can be configured. DS expresses how different parameters and processes relate to optimizing the operating ranges of CPPs.

Finally, a Control Strategy is defined to ensure that the product, whose quality is predefined, is developed and refined within the established parameters [11,14,15,18-22]. At this stage of the process, results obtained from product manufacturing experience can be reintroduced into the QbD methodology, improving quality.

One of the most critical steps for implementing QbD is the configuration of the DS. As mentioned earlier, this process is developed based on the interaction between variables and factors that affect product performance, even before its manufacturing and commercialization [18].

Configuring a DS involves the construction of a surface that represents the quality of a given CPP, as a function of the possible values that it may present (possible operating range) when analyzed considering the guarantee of a specific CQA. Since most of CPPs are distinctly related to multiple CQAs, intersections between different surfaces (the same CPP explored against different CQAs) generate a map that represents the product quality for each value in the potential operating range from this CPP. The analysis of these maps allows the determination of the optimal operating ranges of each $\mathrm{CPP}$, enabling to improve the quality of the development process [23-31].

The benefits achieved with the implementation of QbD principles in the pharmaceutical industry since 2004 [32], and its proactive character, indicate the potential of this tool as a new alternative also for quality assurance of other industrial production modalities [8]. Hence, the adaptation and implementation of $\mathrm{QbD}$ in the development of biomedical devices will possibly provide a significant contribution to the safety and performance assurance of technologies used in the health sector [8].

In alignment with Biometrological precepts [7,8], the Laboratory of Biometrology (LaBioMet) at PUC-Rio conducts research aimed at developing low-cost, highsensitivity biomedical sensors and transducers for safe and noninvasive measurements to be incorporated into the clinical environment. Thus, to contribute to the improvement of the quality assurance of biomedical technologies under development, preliminary initiatives have been undertaken to apply QbD approach in the development of devices for use in healthcare such as assistive technologies [15] and magnetic transducers for biomedical application [16,19]. In these studies, initial steps to configure DS was achieved using Fuzzy Logic.

In Rivero et al. [15], the DS was created by establishing the CPPs associated with the optimal performance of a robotic assistive device to support patients with reduced mobility. By applying a hierarchical Fuzzy model, the complexity of QbD implementation was reduced. Despite the advances, the work evaluated a simplified DS configuration with only two pairs of CPPs associated with two CQAs, being each pair independently analyzed. The subsequent initiative has been the application of the QbD tool in the development of a low-cost magnetic transducer intended for biomedical applications $[16,19]$. This measurement system, under development at LaBioMet, uses magnetometers based on the Giant Magnetoimpedance (GMI) effect, specifically designed to locate non-ferromagnetic metallic objects inserted into the human body. This device is based on a localization technique previously developed by LaBioMet researchers [33], which allows the determination of the position of ferromagnetic foreign object in a noninvasive, innocuous and precise way, allowing to define the surgical approach in order to accelerate and guarantee the successful removal of ferromagnetic foreign bodies [33,34]. Moreover, this methodology based on biomagnetic mapping represents an evolution regarding the alternatives currently available (radiography, computed tomography, and radioscopy), which are not harmless due to the use of ionizing radiation and have a high failure rate in determining the location of foreign objects [33-40]. For locating nonmagnetic metallic objects, such as firearms projectiles, due to the absence of a remnant magnetic field, the measurement system employs a solenoid for primary alternating magnetic field generation to induce eddy currents in the metallic objects. The induced currents generate a secondary magnetic field that can be measured by the high sensitivity magnetic transducer $[35,36,38]$.

These preliminary studies applying the QbD approach in the development of biomedical technologies did not reach a complete analysis of the set of involved parameters $[15,16,19]$, and a greater complexity of the QbD scenario was evidenced when applied to these technological modalities. In these cases, the QbD implementation results in a higher number of parameters (CQAs and CPPs) $[15,16,19]$ when compared to its application in the pharmaceutical industry $[19,22,23,25,26,28-30]$. 
More recently, two other studies investigated the application of QbD approach in the medical field [41,42]. Martinez-Marquez [41] developed an extensive literature review on the suitability of $\mathrm{QbD}$ for the development process of 3D printed custom bone prosthesis and scaffolds. The study was restricted to the first stages of QbD, identifying eight quality dimensions (TPPs) as result of three quality approaches based on the demands of the product, the manufacturing process, and the user. The central parameters constituted of 5 critical quality attributes (CQAs), 11 critical material attributes (CMAs) and 15 critical process parameters (CPPs), were defined to cover the development of a comprehensive design and fabrication process flow diagram and the categorization of the risks associated (risks assessment) with the designing and manufacturing of such products. Despite the linkage established between each CPP and at least one CQA (maximum of four associations), in [41], the design space (DS) was not configured.

Extending previous work, Martinez-Marquez [42] developed a case study in a European firm of the sector of $3 \mathrm{D}$ printed bone implants, to explore the quality control technologies and activities for disseminating industry best practices. This study allowed the adaptation of the QbD approach only through the development of a comprehensive workflow map composed of 27 activities, identifying 16 CPPs, 11 CMAs, as well as 86 risks and 178 effects [42]. However, the study was a qualitative adaptation of QbD and, as a result, did not configure the Design Space, which, in turn, is an essential step of the approach for providing a better performance understanding of the fabrication process.

Thus, the literature does not provide a solution for the configuration of a DS under the interaction of multiple CPPs and CQAs yet, also persisting the challenge of determining the procedure to follow when more than two CPPs have a relationship of dependence with one or more CQAs.

The application of QbD concepts in the development process of a non-ferromagnetic foreign body localization system could provide a significant contribution to guarantee the safety and performance of this technology developed for biomedical applications. However, for the proper implementation of this quality tool in the development process of devices with biomedical applications, one must overcome the challenge of QbD implementation in this field. It is necessary to develop a method that allows the configuration of DS in situations involving a significant number of CQAs and CPPs, which employ several measurement units and operating ranges [16,19].

This work aims at contributing to the adaptation of the QbD methodology to enable its application to ensure the metrological reliability of biomedical technologies. It searches for the overcoming of the notable complexity for implementation of $\mathrm{QbD}$ in this field when compared to the conventional applications of $\mathrm{QbD}$ in the pharmaceutical industry. In this way, this study develops a methodology for analyzing the interaction of multiple CPPs and CQAs, in a multi-parametric environment, using a Fuzzy Inference procedure to configure a DS that meets both the CQAs and the sets of specifications of the device's operating condition. By applying the developed methodology, the present work proceeds and concludes previous researches $[16,19]$. It completes the adaptation of the QbD approach for application in the development phase of a magnetic measuring system for locating non-magnetic metallic foreign objects in patients using low-cost, highsensitivity GMI sensors.

\section{Hybrid QbD-Fuzzy approach implementation}

After a brief description of essential aspects of the biomedical technology under development, this section presents the procedures for identifying the parameters from the first stages of QbD and developing the hybrid QbDFuzzy approach for multiparametric Design Space configuration.

\subsection{Measuring system for nonmagnetic metallic foreign bodies localization under development}

A technique developed and successfully applied for locating ferromagnetic needles for surgical removal employed Superconducting Quantum Interference Device (SQUID) magnetometers [33], which is the most sensitive magnetic sensor, but presents the drawback of requiring liquid helium cooling. In order to comply with biometrological requirements $[7,8]$, magnetometers based on the Giant Magnetoimpedance (GMI) effects were used as lower cost alternatives to SQUID sensors [38].

Previous works presented several characteristics of the magnetic transducer under development [16,19,36-38]; however, the following briefly describes its operation process in the light of the proposed QbD approach implementation.

The operating principle of the magnetic measuring system, designed to locate nonmagnetic metallic foreign bodies, employs a solenoid for primary AC magnetic field generation, which induces eddy currents in metallic objects [35]. In turn, these induced currents produce a timevarying and ultra-low secondary magnetic flux density that can be measured by high-sensitivity GMI sensors arranged in a gradiometric configuration $[35,36,38]$.

The diagram shown in Figure 1 illustrates the solenoid with the two GMI sensors in gradiometric configuration, and a metallic foreign body represented as " $x$ ".

The differential reading performed between the two GMI sensor elements improves the signal-to-noise ratio (SNR) [36-38,43], minimizing the interferences from environmental and primary magnetic flux densities [35-38].

The foreign body location is provided by solving the inverse problem of the magnetic mappings produced by the secondary magnetic flux density generated by the eddy currents induced in the metallic object.

\subsection{Definition of the TPPs, CQAs and CPPs}

The QbD approach uses a systematic analysis between different characteristics of the product (TPP), its quality attributes (CQA), and process parameters (CPP). These 


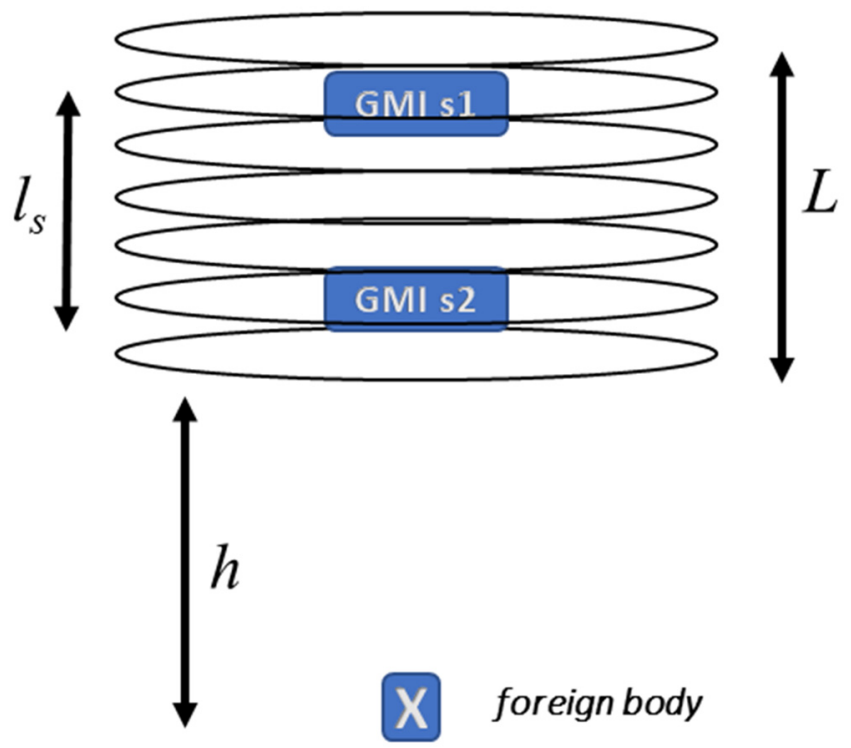

Fig. 1. Solenoid representation with two GMI sensors in gradiometric configuration and a foreign body " $\mathrm{x}$ " [36-38]. $h$ is the distance between the bottom of the solenoid and the foreign object; $L$ is the length of the solenoid; and $l s$ is the distance between the centers of the two GMI sensors (baseline).

parameters were listed after several discussing meetings with the researchers from the Biometrology Laboratory of PUC-Rio (LaBioMet) involved in the magnetic measuring system development. Initial meetings carried out a brainstorm in order to define all the main functionalities and characteristics of the biomedical technology (TPP), considering the biometrological precepts [7,8], aiming to guarantee the safety and appropriate performance, using a metrologically reliable transducer.

Subsequent meetings were held following the same methodology to define the CQA and CPP parameters. The CQA was specified based on the defined TPP, according to the research experts on the transducer's development. The identified CQAs correspond to the system's attributes, which must be controlled during development to ensure the device's operational quality;

In respect to the $\mathrm{CPPs}$, they were identified as the process parameters with a significant influence on CQAs. Among the listed CPPs, only a few of them had their numerical values within an acceptable range, while the others need to operate in a fixed specification. For those CPPs for which a range of values was associated, their degree of influence in relation to each CQA was established. For this purpose, a risk analysis was conducted with which the functional relationships between CPP to CQA were constructed, classifying as high $(\mathrm{H})$, medium $(\mathrm{M})$ or low $(\mathrm{L})$, according to the degree of influence.

\subsection{Hybrid QbD-Fuzzy approach for multiparametric DS configuration}

In the present work, the Design Space (DS) is a twodimensional space formed by orthogonal axes, represented

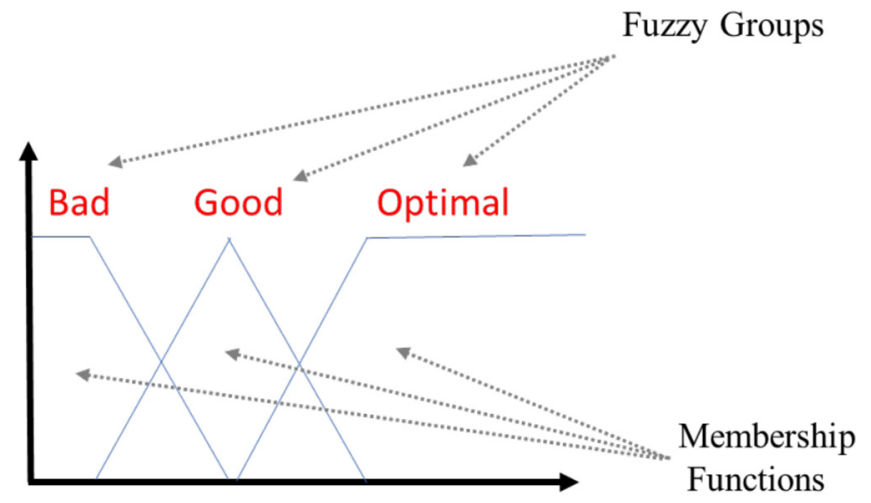

Fig. 2. Representation of the Fuzzy groups by their membership functions.

(each) by a CPP. The QbD approach implementation in the development of a biomedical technology deals with the larger numbers of CQAs and CPPs interacting, associated with the absence of models capable of describing a multiparametric Design Space (DS). Thus, it is proposed a hybrid QbD-Fuzzy methodology that facilitates a complex system's analysis without the need for sophisticated statistical models.

\subsubsection{Fuzzy Inference System (FIS)}

Fuzzy logic became popular from the first studies developed by Zadeh [44], in 1965. This manuscript observed that systems automation in industrial activities, biological or chemical, in which ambiguous situations are inherently present, are impossible to be performed through Boolean logic. Thus, it sought to provide a mathematical tool for the treatment of inaccurate or uncertain information, giving rise to Fuzzy Logic and the systems that use this new form of logic, the Fuzzy Systems $[45,46]$.

All Fuzzy systems can be represented by Fuzzy sets, Fuzzy rules and the defuzzification method. A fuzzy set (or Fuzzy group) represent a linguistic variable and has its elements associated to it by degrees of membership that can assume any value from 0 to 1 . It allows a numeric variable to be represented by the degree of membership in respect to one or more Fuzzy sets, with different membership degrees. Figure 2 shows a representation of the Fuzzy groups and its membership functions associated.

All variables in a Fuzzy inference system are transformed into a linguistic variable (fuzzification) by its representation in the Fuzzy groups. In that way, an input variable can be associated with an adjective like good, bad, high, or low, being each of these a Fuzzy set itself.

The aggregated knowledge in a Fuzzy system is condensed in a set of rules (Fuzzy rules), which characterize the inference goals.

The Fuzzy rules are organized as a linguistic sentence that establishes a causality relationship between one or more input variables and the output variable. These linguistics variables are associated with the Fuzzy rules in a sentence like $I F-T H E N$. 
Input 1

Input 2

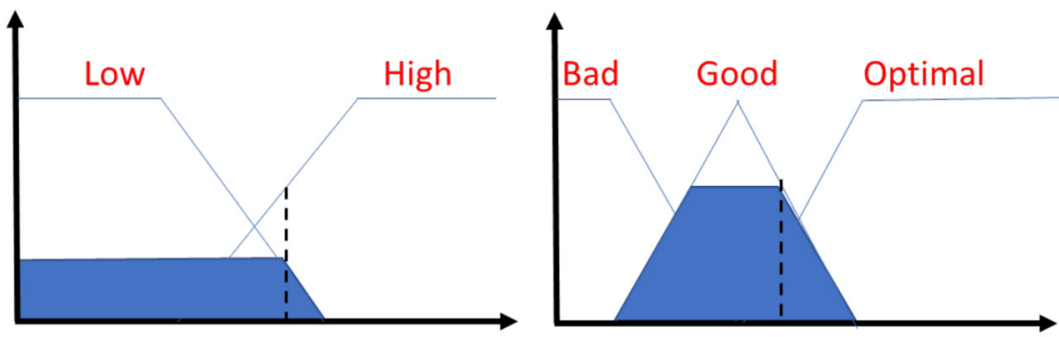

Output

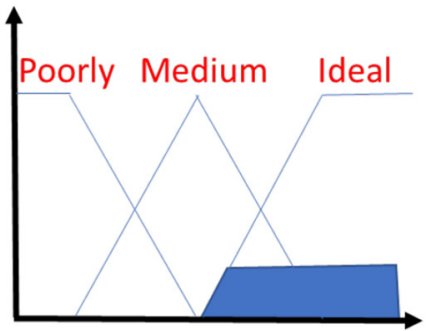

\section{IF input 1 is low AND input 2 is good, THEN output is ideal}

Fig. 3. Representation of two Fuzzy inputs sets and their corresponding Fuzzy output distribution, according to the Fuzzy rule.

As an example, one could write a Fuzzy rule as a sentence like "IF an input variable (called an antecedent) is identified as good or high, THEN output variable (consequent) will be positive or negative". When more than one input variable is considered simultaneously, the linguistic sentences are linked by the linguistic connection of union $(O R)$ or intersection $(A N D)$. Thus, logical sentences can be represented by:

IF, antecedent_1 is (adjective) AND antecedent_2 is (adjective), THEN consequent is (adjective).

As the inference system is performance, the result achieved is given by the activation of some output Fuzzy sets. In Figure 3 is shown the application of a Fuzzy rule and its implication on the Fuzzy input and output sets.

Defuzzification transforms the linguistic output back into a numerical attribute. In other words, it converts the information associated with the linguistic output variable into a numerical value that can be evaluated by the user [45-47]. This method analyzes the activated output Fuzzy sets with the degree of membership established by the input to extract crisp numerical information.

There are different ways to carry out the defuzzification process. Among the most common are the average of the maximum (MoM), the centroid (center-of-gravity), the smallest of maximum (SoM), and the largest of maximum (LoM) [47]. For example, when applying the centroid defuzzification method, it calculates the centroid of the geometric figure formed by the activated outputs Fuzzy sets. The returned number is the output of the Fuzzy inference system. Figure 4 illustrates the defuzzification results using the centroid method. After applying all the Fuzzy rules, it results in the activation of several output Fuzzy sets.

The present work applied the LoM defuzzification method for the DS configuration of the evaluated CPPs to achieve the highest level of adequacy in each critical parameter's operating ranges.

\subsubsection{QbD-Fuzzy adaptation}

In this paper, after defining the set of CPPs related to each CQA, a risk analysis was carried out to select the parameters to be analyzed. Thus, only CPPs with a high impact on the related CQAs were considered as Fuzzy linguistic variables.

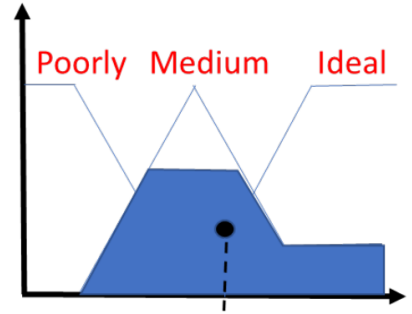

Fig. 4. Representation of the output Fuzzy sets resulting from a centroid defuzzification.

The QbD-Fuzzy Inference System stages for DS configuration used in this work are shown in Figure 5. The first step in the Fuzzy inference is the fuzzification of each CPP selected in the risk analysis. Here the numerical values of the CPP are associated with a Fuzzy set.

Triangular and trapezoidal shapes represented the membership functions employed. They were classified into three output groups, namely, "Optimal" (optimal level of performance of the variable), "Good" (good level of performance of the variable), and "Bad" (low level of performance of the variable). The adequacy of the operating ranges of the CPP values in correspondence to each associated CQA was identified. The membership functions' configuration for each linguistic variable (CPPs) was carried out with the Toolbox "Membership Function" of the Matlab R2011b software.

The Fuzzy rules were defined according to the relationship of each Fuzzy set corresponding to each variable and their interaction with the relevant CQAs.

The risk analysis between CQAs and CPPs carried out as a preliminary step to the DS configuration determined the dependency relationship between these parameters according to the product profile. This relationship was established considering the degree of influence of each CPP in the several CQAs identified. This degree being classified as high $(\mathrm{H})$, medium (M) or low (L). Only parameters classified with high impact $(\mathrm{H})$ were selected for the DS configuration.

Thus, all possible cases of CPPs related to one or more CQAs were identified. A Fuzzy inference model represents each interaction between a given CPP and the CQAs it influences, where each CPP's operating range varies with 


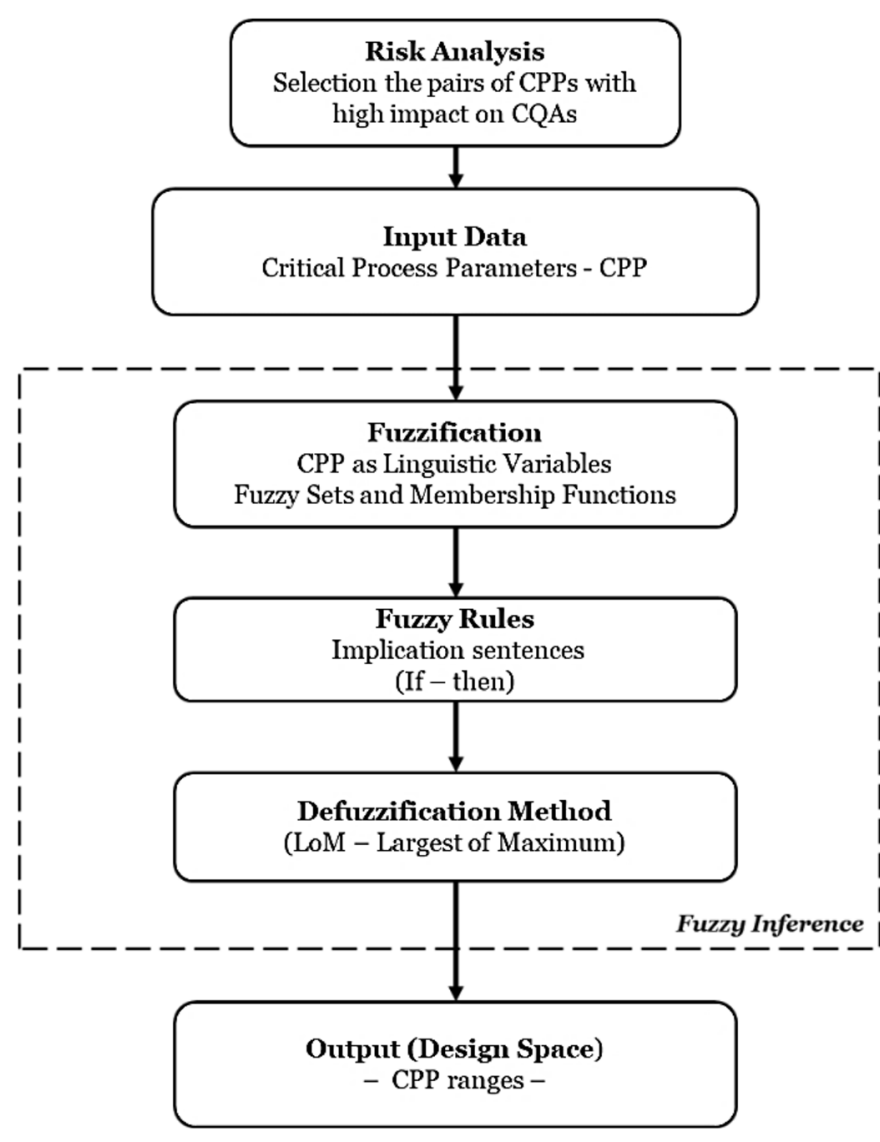

Fig. 5. Fuzzy inference steps for developing the Design Space.

the associated CQA. Therefore, it is possible to obtain an adequate operating space for this critical parameter (CPP) regarding a CQA it influences. The Fuzzy maps generated by this CPP's interaction with the CQAs represent the CPP's DS, which informs its best operating range.

Once the DS was configured by simulating the behavior of the critical parameters (CPPs) associated with each CQA, a control strategy was established to ensure the successful implementation of QbD. This strategy focused on carrying out a qualitative control based on the verification of the TPPs' alignment with the CPPs and CQAs resulting from the risk analysis carried out.

\section{Results}

This section presents the QbD parameters identified, considering the essential aspects associated with the biomedical technology under development, and describes the results obtained in the implementation of the proposed hybrid QbD-Fuzzy approach for multiparametric Design Space configuration.

\subsection{Identification of the TPPs, CQAs and CPPs}

Tables 1 and 2 presents, respectively, the Target Product Profile (TPP) and the Critical Quality Attributes (CQA)
Table 1. Target product profile (TPP) associated with the measuring system for biomedical application.

\begin{tabular}{ll}
\hline Coding & Target product profile (TPP) \\
\hline $\mathrm{TPP}_{1}$ & High precision and accuracy \\
$\mathrm{TPP}_{2}$ & Low measurement uncertainty \\
$\mathrm{TPP}_{3}$ & Safety \\
$\mathrm{TPP}_{4}$ & Low complexity in manufacturing \\
$\mathrm{TPP}_{5}$ & Low manufacturing cost \\
$\mathrm{TPP}_{6}$ & Low operating cost \\
$\mathrm{TPP}_{7}$ & Low complexity of operation \\
$\mathrm{TPP}_{8}$ & Portability \\
$\mathrm{TPP}_{9}$ & Low maintenance complexity \\
$\mathrm{TPP}_{10}$ & (calibration included) \\
\hline
\end{tabular}

Table 2. CQA associated with the measurement system for biomedical application.

\begin{tabular}{ll}
\hline Coding & Critical quality attributes (CQA) \\
\hline $\mathrm{CQA}_{1}$ & Spatial resolution $(\mathrm{mm})$ \\
$\mathrm{CQA}_{2}$ & Operation at room temperature (yes/no) \\
$\mathrm{CQA}_{3}$ & Transducer sensitivity $(\mathrm{mV} / \mu \mathrm{T})$ \\
$\mathrm{CQA}_{4}$ & Signal-to-noise ratio $(\mathrm{dB})$ \\
$\mathrm{CQA}_{5}$ & Secondary magnetic field strength $(\mathrm{nT})$ \\
$\mathrm{CQA}_{6}$ & Temporal resolution $(\mu \mathrm{s})$ \\
\hline
\end{tabular}

that, should characterize the biomedical technology under development, which is a measuring system based on a high sensitivity GMI magnetic transducer, for locating non-magnetic metallic foreign bodies in the human organism. These priority features and attributes, designed according to biometrological principles [7,8], consider the characteristics that ensure a portable and affordable technology with safety and proper performance, thus promoting efficient treatment and diagnosis, and avoiding adverse events.

Table 3 lists the 19 Critical Process Parameters (CPP) defined for the biomedical measuring device. The seven CPPs highlighted in bold in Table $3\left(\mathrm{CPP}_{1}, \mathrm{CPP}_{2}, \mathrm{CPP}_{3}\right.$, $\mathrm{CPP}_{6}, \mathrm{CPP}_{14}, \mathrm{CPP}_{17}$ and $\mathrm{CPP}_{18}$ ) were selected for the application of the Fuzzy inference. The remaining 12 CPPs are associated with a single acceptable specification or fixed value, for which there is no range.

\subsection{Risk analysis for DS configuration}

The interaction between the seven selected CPPs, in bold in Table 3, and the six CQAs, listed in Table 2, was assessed by conducting a risk analysis, which provided information on the degree of influence that a given CPP has on each CQA. In turn, the adequacy of the CQA attributes reflects in the fulfilling of requirements necessary to reach the characteristics associated with the listed TPPs. Table 4 
Table 3. Critical process parameters (CPPs) associated with the measurement system for biomedical application.

\begin{tabular}{|c|c|}
\hline Coding & Critical process parameters (CPPs) \\
\hline $\mathbf{C P P}_{1}$ & Primary magnetic field frequency $(\mathrm{kHz})$ \\
\hline $\mathrm{CPP}_{2}$ & Current intensity (solenoid excitation) (mA) \\
\hline $\mathrm{CPP}_{3}$ & Current intensity (circuit) (mA) \\
\hline $\mathrm{CPP}_{4}$ & Circuit supply voltage (V) \\
\hline $\mathrm{CPP}_{5}$ & Sensor sensitivity $(\mathrm{mV} / \mathrm{nT})$ \\
\hline $\mathrm{CPP}_{6}$ & Solenoid dimensions $\left(\mathrm{N} / \mathbf{L}^{*}\right.$ ratio) (turns $\left./ \mathbf{m}\right)$ \\
\hline $\mathrm{CPP}_{7}$ & GMI supply voltage (V) \\
\hline $\mathrm{CPP}_{8}$ & Oscillator supply voltage $(\mathrm{V})$ \\
\hline $\mathrm{CPP}_{9}$ & Sensor characteristics (intrinsic noise) $(\mathrm{fT} / \sqrt{ } \mathrm{Hz})$ \\
\hline $\mathrm{CPP}_{10}$ & $\mathrm{AC}$ in the GMI sensor (intensity) (mA) \\
\hline $\mathrm{CPP}_{11}$ & $\mathrm{AC}$ frequency of the current in the sensor (oscillator frequency) (MHz) \\
\hline $\mathrm{CPP}_{12}$ & Sensor size (length) $(\mathrm{cm})$ \\
\hline $\mathrm{CPP}_{13}$ & Heterogeneity of sensors (difference in sensitivity) (\%) \\
\hline $\mathrm{CPP}_{14}$ & Gradiometer baseline (cm) \\
\hline $\mathrm{CPP}_{15}$ & Voltage stability (voltage source) (\%) \\
\hline $\mathrm{CPP}_{16}$ & Signal amplification (circuit) $(\mathrm{V} / \mathrm{V})$ \\
\hline $\mathbf{C P P}_{17}$ & $\begin{array}{l}\text { Superposition of baseline lengths between sensor centers and solenoid axis } \\
\text { (solenoid length \%) }(\mathrm{cm})\end{array}$ \\
\hline $\mathrm{CPP}_{18}$ & Circuit noise (intrinsic noise) (pT) \\
\hline $\mathrm{CPP}_{19}$ & Filter (order) \\
\hline
\end{tabular}

$*_{\mathrm{N}} / \mathrm{L}=$ Number of turns per unit length of the solenoid. $\mathrm{N}=36$ turns, $\mathrm{L}=0.07 \mathrm{~m}$.

Table 4. Risk analysis to classify the degree of influence between CQAs and CPPs as high (H), medium (M), or low (L) impact.

\begin{tabular}{llllllll}
\hline $\mathrm{CPPs}$ & $\mathrm{CPP}$ & $\mathrm{CPP}$ & $\mathrm{CPP}_{3}$ & $\mathrm{CPP}_{6}$ & $\mathrm{CPP}_{14}$ & $\mathrm{CPP}_{17}$ & $\mathrm{CPP}_{18}$ \\
\hline $\mathrm{CQAs}$ & & & & & & \\
$\mathrm{CQA}_{1}$ & $\mathrm{~L}$ & $\mathrm{~L}$ & $\mathrm{~L}$ & $\mathrm{~L}$ & $\mathrm{H}$ & $\mathrm{L}$ & $\mathrm{H}$ \\
$\mathrm{CQA}_{2}$ & $\mathrm{~L}$ & $\mathrm{~L}$ & $\mathrm{~L}$ & $\mathrm{~L}$ & $\mathrm{~L}$ & $\mathrm{H}$ & $\mathrm{L}$ \\
$\mathrm{CQA}_{3}$ & $\mathrm{H}$ & $\mathrm{H}$ & $\mathrm{H}$ & $\mathrm{H}$ & $\mathrm{H}$ & $\mathrm{H}$ & $\mathrm{H}$ \\
$\mathrm{CQA}_{4}$ & $\mathrm{H}$ & $\mathrm{H}$ & $\mathrm{H}$ & $\mathrm{H}$ & $\mathrm{H}$ & $\mathrm{H}$ & $\mathrm{H}$ \\
$\mathrm{CQA}_{5}$ & $\mathrm{H}$ & $\mathrm{H}$ & $\mathrm{L}$ & $\mathrm{H}$ & $\mathrm{L}$ & $\mathrm{L}$ & $\mathrm{L}$ \\
$\mathrm{CQA}_{6}$ & $\mathrm{H}$ & $\mathrm{L}$ & $\mathrm{L}$ & $\mathrm{L}$ & $\mathrm{M}$ & $\mathrm{H}$ & $\mathrm{L}$ \\
\hline
\end{tabular}

shows the levels of influence between CPPs and CQAs, established by the LaBioMet researchers involved in the biomedical technology development.

In the risk analysis's brainstorming, it was assumed that only the pairs of CPPs and CQAs with a strong dependency relationship would have a deeper relevance on the transducer quality. It was also realized that the CPPs were not susceptible to variations from other CPPs. Thus, the only quantities that directly impact the operating ranges of a given CPP are the CQAs with a high rating $(\mathrm{H})$ with this CPP. This aspect has greatly reduced the complexity of adapting de DS configuration.

The parameter relationship, shown in Table 4, indicates that each CPP significantly affects at least two and at most four of the six CQAs. In the specific case of $\mathrm{CQA}_{3}$ and $\mathrm{CQA}_{4}$ attributes, these are impacted by all the seven CPPs. For $\mathrm{CQA}_{2}$, however, only one $\mathrm{CPP}$ was considered relevant. This information is used for constructing the DS for these seven CPPs, as described in the next section.

\subsection{QbD-FIS for multiparametric DS configuration}

For the biomedical technology under development, a given CPP may have different appropriate values when analyzed against different CQAs. Hence, the operating ranges of each CPP parameter suitable for each CQA attribute have been identified. 


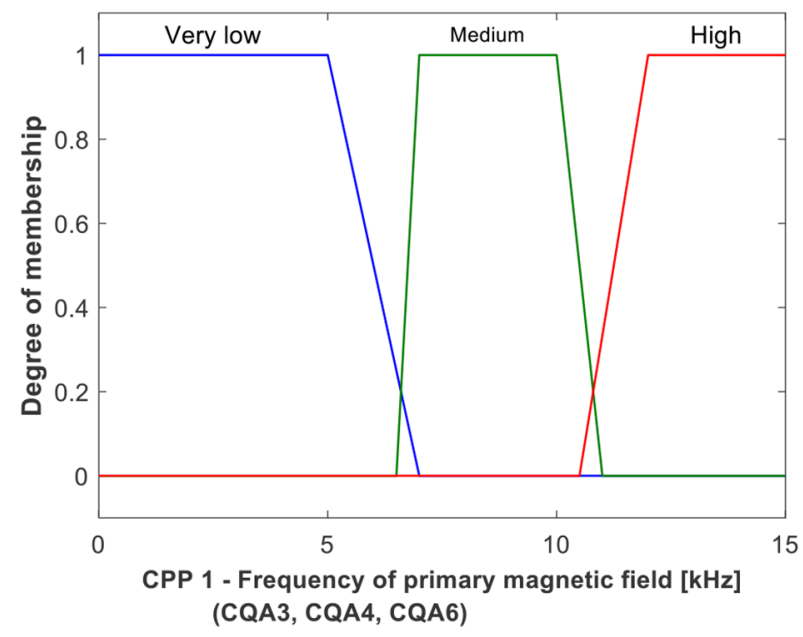

(a)

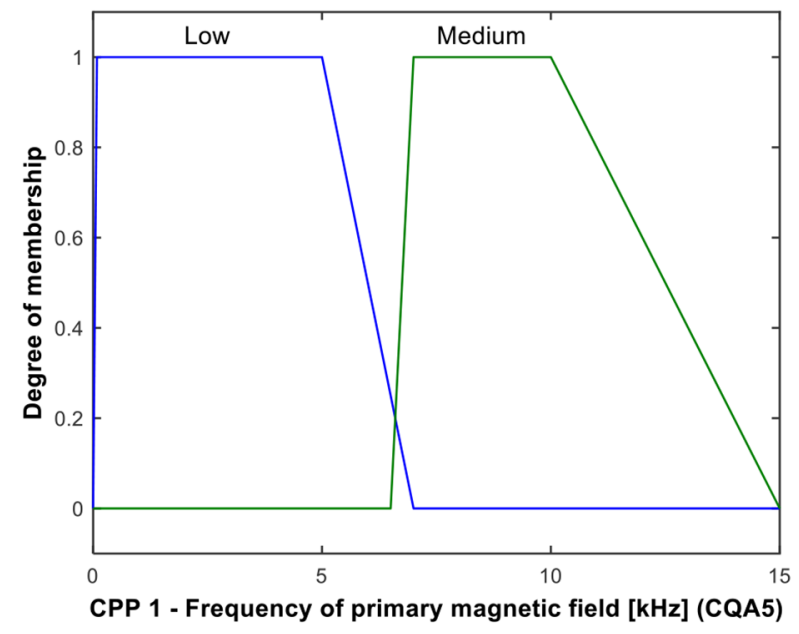

(b)

Fig. 6. Membership function for $\mathrm{CPP}_{1}$. (a) Fuzzy input $1-\mathrm{CPP}_{1}$ related to $\mathrm{CQA}_{3}, \mathrm{CQA}_{4}, \mathrm{CQA}_{6}$; (b) Fuzzy input $2-\mathrm{CPP}_{1}$ related to $\mathrm{CQA}_{5}$.

To represent the Fuzzy inference of the interaction between a given CPP and the CQAs to which it influences, two-dimensional maps were generated. Thus, for a given critical process parameter, each axis of the map represents the dependency of a given CQA or group of CQAs on the operating range of this CPP. Seven Fuzzy inference systems were employed to build the multiparametric design space related to the biomedical measuring system under development. These systems are associated with each of the seven selected CPPs (highlighted in bold, in Tab. 3), constituted by $\mathrm{CPP}_{1}, \mathrm{CPP}_{2}, \mathrm{CPP}_{3}, \mathrm{CPP}_{6}, \mathrm{CPP}_{14}$, $\mathrm{CPP}_{17}$ and $\mathrm{CPP}_{18}$.

For each input linguistic variables represented by the seven CPPs, 29 Fuzzy sets of membership functions were built according to their suitability to the CQAs with which they have higher dependency relationship. The membership functions indicated that $\mathrm{CPP}_{1}, \mathrm{CPP}_{2}, \mathrm{CPP}_{3}$, and $\mathrm{CPP}_{6}$ presented at least two different CQAs dependence range. On the other hand, the other three process parameters $\left(\mathrm{CPP}_{14}, \mathrm{CPP}_{17}\right.$ and $\left.\mathrm{CPP}_{18}\right)$ presented the same input Fuzzy set associated with suitable values for different CQAs. In this case, the Fuzzy inference will return not a two-dimensional map, but a unidimensional graphic.

For sake of space, the two fuzzification conditions are exemplified in Figure 6, for $\mathrm{CPP}_{1}$, representing the CPPs with two different membership function $\left(\mathrm{CPP}_{1}, \mathrm{CPP}_{2}\right.$, $\mathrm{CPP}_{3}$, and $\mathrm{CPP}_{6}$ ); and in Figure 7 , for $\mathrm{CPP}_{14}$, representing the CPPs with a single relevant input Fuzzy set $\left(\mathrm{CPP}_{14}\right.$, $\mathrm{CPP}_{17}$ and $\left.\mathrm{CPP}_{18}\right)$.

The fuzzification with two different Fuzzy input sets related to the $\mathrm{CPP}_{1}$, which corresponds to the frequency of primary magnetic field necessary to induce eddy currents in the metallic foreign body to be localized, are presented in Figure $6 \mathrm{a}$ for membership function associated with the $\mathrm{CQA}_{3}, \mathrm{CQA}_{4}$, and $\mathrm{CQA}_{6}$; and, in Figure 6b, considering the proper $\mathrm{CPP}_{1}$ values for $\mathrm{CQA}_{5}$.

The fuzzification exemplified for CPPs with which a single input Fuzzy set is associated is presented for in

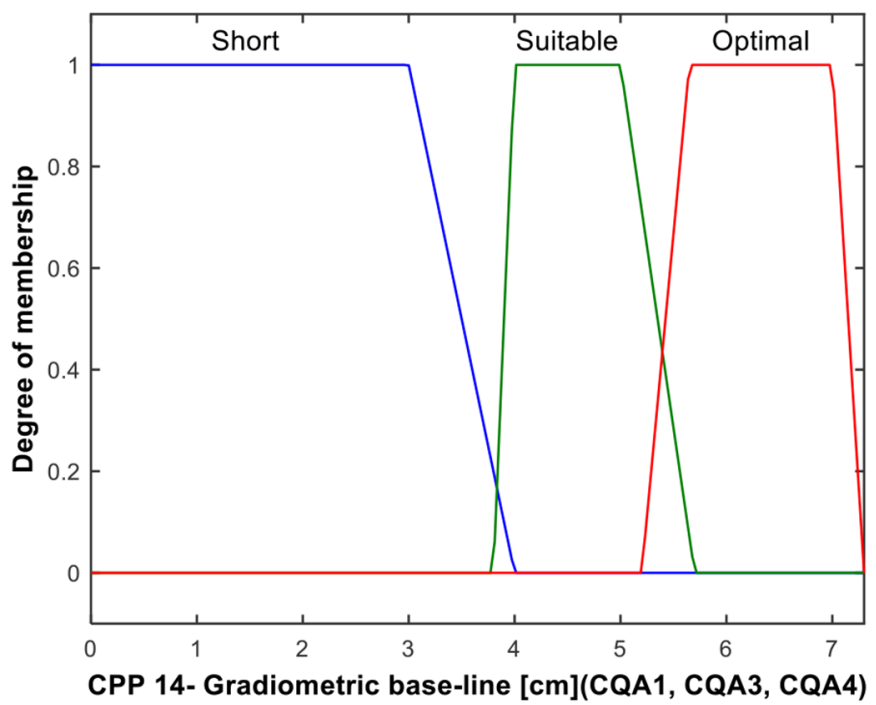

Fig. 7. Fuzzy sets of the $\mathrm{CPP}_{14}$.

Figure 7, for $\mathrm{CPP}_{14}$, which corresponds to the gradiometer baseline, indicating the appropriate distance between the GMI sensors. The same Fuzzy sets are associated with the three attributes $\left(\mathrm{CQA}_{1}, \mathrm{CQA}_{3}\right.$ and $\left.\mathrm{CQA}_{4}\right)$ affected by $\mathrm{CPP}_{14}$.

Table 5 presents the Fuzzy rules, associated with the exemplified CPP results of Figure 6 (for $\mathrm{CPP}_{1}$ ) and Figure 7 (for $\mathrm{CPP}_{14}$ ).

The results comprise seven Fuzzy inference systems employed for Design Space configuration. Figures 8 and 9 exemplifies in detail the two possible output CPP behaviors of the graphical implementation of DS. These two Fuzzy inference systems are detailed for the CPP parameters for which the membership functions are presented in Figure $6\left(\mathrm{CPP}_{1}\right)$ and Figure $7\left(\mathrm{CPP}_{14}\right)$, and Fuzzy rules are described in Table $5\left(\mathrm{CPP}_{1}\right.$ and $\left.\mathrm{CPP}_{14}\right)$. 
Table 5. Fuzzy rules for $\mathrm{CPP}_{1}$ and $\mathrm{CPP}_{14}$.

$\mathrm{CPP}_{1}$

IF CPP1 (CQA3, CQA4, CQA6) is very low AND CPP1 (CQA5) is Low, THEN output for CPP1 is bad IF CPP1 (CQA3, CQA4, CQA6) is medium AND CPP1 (CQA5) is Low, THEN output for CPP1 is good IF CPP1 (CQA3, CQA4, CQA6) is high AND CPP1 (CQA5) is Low, THEN output for CPP1 is bad

IF CPP1 (CQA3, CQA4, CQA6) is very low AND CPP1 (CQA5) is medium, THEN output for CPP1 is good

IF CPP1 (CQA3, CQA4, CQA6) is medium AND CPP1 (CQA5) is medium, THEN output for CPP1 is optimal

IF CPP1 (CQA3, CQA4, CQA6) is high AND CPP1 (CQA5) is medium, THEN output for CPP1 is good

$\mathrm{CPP}_{14}$

IF CPP14 (CQA1, CQA2, CQA4) is short, THEN output for CPP14 is bad

IF CPP14 (CQA1, CQA2, CQA4) is suitable, THEN output for CPP14 is bad

IF CPP14 (CQA1, CQA2, CQA4) is optimal, THEN output for CPP14 is optimal

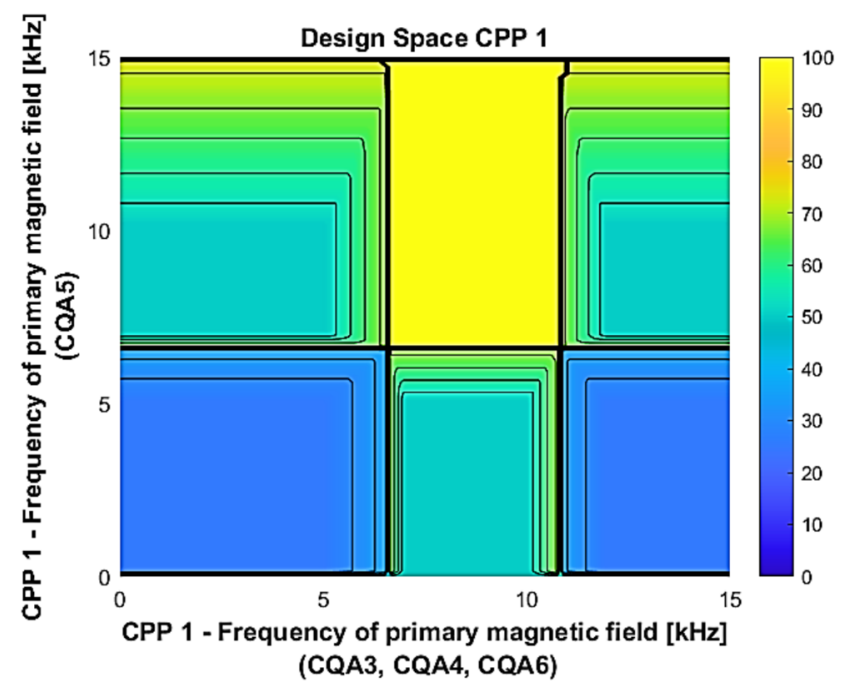

(a)

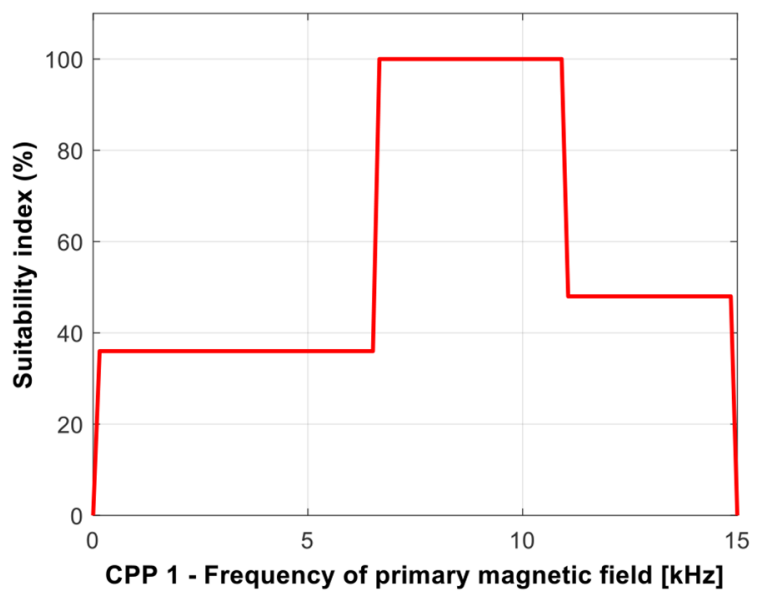

(b)

Fig. 8. Design Space map obtained for $\mathrm{CPP}_{1}$, in (a); range of appropriate values of $\mathrm{CPP}_{1}$ for optimum performance of the measurement system, in (b).

Concluding results for the Design Space configuration consider all the relevant CPPs.

The graphical correlation between the operating ranges of each CPP and its adequacy to the CQAs associated indicates the acceptable operating space of the CPP for which the device reaches its maximum effectiveness, constituting the Design Space of the process parameter.

Figure 8 presents the output of the Fuzzy inference system for $\mathrm{CPP}_{1}$. The two-dimensional map corresponds to the Design Space of this parameter (Fig. 8a), in which the " $X$ " axis shows its relationship with $\mathrm{CQA}_{3}, \mathrm{CQA}_{4}$, and $\mathrm{CQA}_{6}$, while the " $Y$ " axis shows the relationship with the $\mathrm{CQA}_{5}$.

The suitability of the $\mathrm{CPP}_{1}$ values for the proper operation of the device under development is indicated using a color scale from 0 to 100 (Fig. 8a), informing the correspondence between the parameter value and its suitability. The intersection between the maximum levels reached on each axis of Figure 8a allows identifying an index of performance adequacy for each $\mathrm{CPP}_{1}$ value (Fig. $8 \mathrm{~b}$ ). The analysis of Figure $8 \mathrm{~b}$ indicates that the

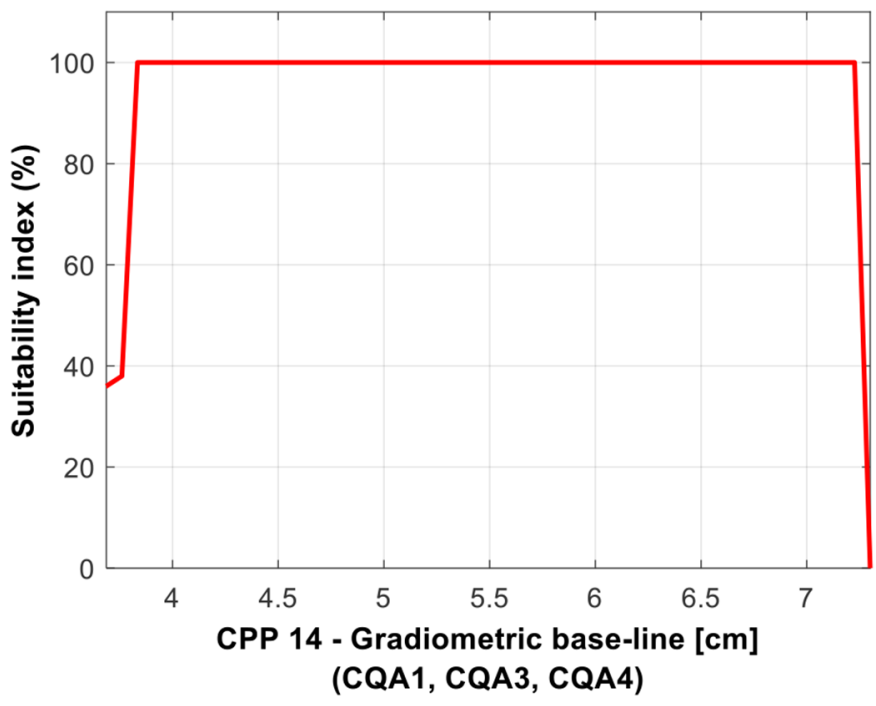

Fig. 9. Suitability index of $\mathrm{CPP}_{14}$ according to values of baseline distance between GMI sensors. 
Table 6. Suitable CPP operating ranges that guarantee the best performance of the device.

\begin{tabular}{|c|c|c|c|}
\hline Code & Critical Process Parameters (CPP) & Suitable operating ranges & Prototype device \\
\hline $\mathrm{CPP}_{1}$ & Primary magnetic field frequency & $6.7-10.9 \mathrm{kHz}$ & $7.7 \mathrm{~Hz}$ \\
\hline $\mathrm{CPP}_{2}$ & Current intensity (solenoid excitation) & $1.6-2.09 \mathrm{~mA}$ & $1.8-2.0 \mathrm{~mA}$ \\
\hline $\mathrm{CPP}_{3}$ & Current intensity (circuit) & $129.3-387.9 \mathrm{~mA}$ & $\sim 100 \mathrm{~mA}$ \\
\hline $\mathrm{CPP}_{4}$ & Circuit supply voltage (V) & - & $\pm 15 \mathrm{~V}$ \\
\hline $\mathrm{CPP}_{5}$ & Sensor sensitivity $(\mathrm{mV} / \mathrm{nT})$ & - & $5 \mathrm{mV} / \mathrm{nT}$ \\
\hline $\mathrm{CPP}_{6}$ & Solenoid dimensions $\left(\mathrm{N} / \mathrm{L}^{*}\right.$ ratio $)$ & 505-1 000 turns $/ \mathrm{m}$ & $\sim 514.3$ turns $/ \mathrm{m}$ \\
\hline $\mathrm{CPP}_{7}$ & GMI supply voltage $(\mathrm{V})$ & - & $15 \mathrm{~V}$ \\
\hline $\mathrm{CPP}_{8}$ & Oscillator supply voltage (V) & - & $\sim 5 \mathrm{~V}$ \\
\hline $\mathrm{CPP}_{9}$ & Sensor characteristics (intrinsic noise) $(\mathrm{fT} / \sqrt{ } \mathrm{Hz})$ & - & $10 \mathrm{fT} / \sqrt{ } \mathrm{Hz}$ \\
\hline $\mathrm{CPP}_{10}$ & $\mathrm{AC}$ in the GMI sensor (intensity) (mA) & - & $\sim 15 \mathrm{~mA}$ \\
\hline $\mathrm{CPP}_{11}$ & $\begin{array}{l}\text { AC frequency of the current in the sensor } \\
\text { (oscillator frequency) }(\mathrm{MHz})\end{array}$ & - & $1 \mathrm{MHz}$ \\
\hline $\mathrm{CPP}_{12}$ & Sensor size (length) $(\mathrm{cm})$ & - & $1.35 \mathrm{~cm}$ \\
\hline $\mathrm{CPP}_{13}$ & Heterogeneity of sensors (difference in sensitivity) (\%) & - & $<1 \%$ \\
\hline $\mathrm{CPP}_{14}$ & Gradiometer baseline & $3.8-7.2 \mathrm{~cm}$ & $\sim 5.65 \mathrm{~cm}$ \\
\hline $\mathrm{CPP}_{17}$ & $\begin{array}{l}\text { Superposition of baseline lengths between } \\
\text { sensor centers and solenoid axis (\% of solenoid's length) }\end{array}$ & up to $6.6 \% \mathrm{~cm}$ & up to $2 \% \mathrm{~cm}$ \\
\hline $\mathrm{CPP}_{18}$ & Circuit noise (intrinsic noise) & up to $37.4 \mathrm{pT}$ & $25 \mathrm{pT}$ \\
\hline $\mathrm{CPP}_{19}$ & Filter (order) & - & fourth-order \\
\hline
\end{tabular}

frequency of the primary magnetic field $\left(\mathrm{CPP}_{1}\right)$ must be within a range of 6.7 to $10.9 \mathrm{kHz}$ to ensure the optimum device's performance, i.e., a suitability index of $100 \%$.

Figure 9 presents the results of Design Space for $\mathrm{CPP}_{14}$, which exemplifies a unidimensional parameter behavior, to which a single input Fuzzy set is associated. In this case, the output directly represents the suitability index. The results indicate that the baseline of the gradiometer $\left(\mathrm{CPP}_{14}\right)$ should be in a range from 3.8 to $7.2 \mathrm{~cm}$, to guarantee the ideal performance of the device ( $100 \%$ adequacy index).

Table 6 shows the complete results for the 19 CPPs. The table lists the most suitable operational specification, value or range of the process parameter for which the biomedical technology for locating foreign objects in the human body operates efficiently.

\section{Discussion}

This work presents the adaptation of QbD approach for its complete implementation in the developing stage of a biomedical technology, by incorporating Fuzzy inference systems. The study develops a methodology for analyzing the interaction of multiple CPPs and CQAs, in a multiparametric environment, using a Fuzzy Inference procedure to configure a DS that meets both the CQAs and the sets of specifications of the device's operating condition. By applying the developed methodology, the present work proceeds and concludes previous preliminary researches $[16,19]$.

The developed methodology based on the QbD concepts in conjunction with the Fuzzy logic was implemented in a magnetic measuring system under development at LaBioMet, which uses low-cost and high-sensitive magnetometers based on the Giant Magnetoimpedance effect and is designed to locate nonferromagnetic metallic objects inserted into the human body. The resulting magnetic mapping provided by the technology can be a guide for defining the strategy for successful surgical removal, as already been demonstrated in the literature using higher-cost sensors, based on superconductivity [33].

The list of TPPs defined for the device comprised ten features based on the technology's functionalities that guarantee its application and the fulfillment of biometrological precepts $[7,8]$.

Some of the relevant biometrological precepts that guided the development of this research were $[7,8]$ :

- Innocuousness - In the clinical environment, the location of metallic foreign bodies is based on techniques that are characterized by the exposure of patients and staff to high levels of ionizing radiation. The measurement system under development is designed for performing the localization by measuring the magnetic flux density generated by currents induced in the foreign body, respecting the acceptable levels of exposure to nonionizing radiation, according to the guidelines published by the International Commission on Non-Ionizing Radiation Protection (ICNIRP) [48].

- Non-invasiveness - The location of non-magnetic metallic foreign objects, by measuring the magnetic flux density is intrinsically a non-invasive procedure.

- Low complexity and low cost - The use of sensors based on the GMI property, capable of measuring, at room temperature, low levels of magnetic flux density (nT), have low manufacturing and operating costs when 
compared to other high-performance sensitivity systems, such as SQUID (Superconducting Quantum Interference Device), which require cryogenic temperatures for operation.

- Safety - The general requirements for the safety and performance of electro-medical equipment are provided internationally by the IEC (International Electrotechnical Commission) and ISO (International Organization for Standardization) standards, being incorporated as requirements by regulations published by National Health Surveillance Agencies. Regarding specific safety and performance requirements for medical equipment dedicated to magnetic field measurements, there are still no specific technical standards available by standardization bodies. In any case, the measurement system using a GMI transducer under development uses low levels of current and electrical voltage, which simplifies the compliance with electrical safety requirements.

- Portability - In addition to operating at room temperature, the location system being developed is a portable device and can be easily transported and used in different clinical environments. The total size of the GMI system depends mainly on the size of the electronic circuits and the solenoid used for primary magnetic field generation to induce eddy currents in the metallic foreign body to be removed.

Six CQAs and 19 most significant CPPs associated with the quality of the technology under development were identified. The highest dependence relationship parameters were delimited through risk analysis, establishing the largest number of critical combinations for Design Space construction.

Although CPPs are not interdependent, it has been observed that several CQAs are susceptible to variations in multiple CPPs (7 CPPs). In addition, the same CPP can present appropriate ranges of operation with different values for each CQA considered. This difference between ranges of a given CPP for different CQAs was observed in four of the seven CPPs in which the Fuzzy inference was applied: $\mathrm{CPP}_{1}$ (primary magnetic field frequency $[\mathrm{kHz}]$ ), $\mathrm{CPP}_{2}$ (intensity of the solenoid excitation current $[\mathrm{mA}]$ ), $\mathrm{CPP}_{3}$ (circuit current intensity $[\mathrm{mA}]$ ) and $\mathrm{CPP}_{6}$ (dimensions of the solenoid $[\mathrm{N} / \mathrm{L}$ ratio - turns $/ \mathrm{m}])$. The other three CPPs to which Fuzzy inferences were associated, namely, $\mathrm{CPP}_{14}$ (gradiometer baseline $[\mathrm{cm}]$ ), $\mathrm{CPP}_{17}$ (overlapping the baseline lengths between the sensor centers and the solenoid axis [\% of solenoid length]), and $\mathrm{CPP}_{18}$ (circuit noise (intrinsic noise) [pT]) presented only one Fuzzy set of inputs regarding to the related CQAs.

For each combination of CPP with relevant CQAs, an experimental model was structured to determine the behavior of the critical parameters and their best performance ranges. Thus, the DS was configured to represent the most suitable operating ranges of CPPs that satisfy the functionalities of the measuring system under development, assuring its quality.

In the pharmaceutical industry, in turn, for a given CQA, there is a function or dependency between two different CPPs. Although in the development of the biomedical device the best ranges of the CPP parameters did not present interdependence with each other, it proved necessary to assess their suitability for multiple CQAs. Besides, the first attempts to adapt QbD to biomedical devices results in a much higher number of parameters (CQAs and CPPs) $[15,16,19,41,42]$.

In [41] and [42], the first stages of QbD were implemented for 3D printed bone implants, and in [15] for a robotic assistive technology; however, none of these studies worked on the Design Space configuration. The initiatives for implementing the DS stage of QbD for biomedical technologies, developed in [16] and [19], evidenced the challenge of providing a procedure when more than two CPPs have a relationship of dependence with more than one CQA. As shown in Table 4, the complex system includes CQAs associated with seven CPPs. The present work, by implementing Fuzzy Inference Systems, successfully configures the Design Space, characterizing the acceptable operating ranges of the large number of CPPs that optimize the CQAs for each of the measuring system's functionalities (Tab. 6).

\section{Conclusions}

The work presents a complete adaption of the Quality by Design approach to managing the quality of a developing device intended for clinical use. The Design Space configuration was built by incorporating Fuzzy inference systems, overcoming the inherent complexity associated with the high number of critical parameters impacting quality attributes for QbD implementation in biomedical technology development. The proposed approach was applied for the quality specification of a magnetic measuring system to locate non-ferromagnetic metallic foreign bodies in patients, employing high-sensitivity GMI sensors. The low-cost measuring system has the purpose of non-invasive providing foreign objects' position and its projection to the skin, aiming at guiding the medical conduct for successful and quick surgical removal.

The QbD parameters, including 10 TPPs, 6 CQAs, and 19 CPPs, were defined for the biomedical measuring device in compliance with the biometrological precepts, promoting the development of affordable technologies that provide safe and reliable diagnosis and treatment $[7,8]$. The QbD parameters were associated with the Fuzzy logic procedures, and seven Fuzzy Inference Systems were implemented for configuring the multiparametric DS.

The proposed approach reduced data processing complexity in determining the acceptable operating ranges of the large number of CPPs that optimize the CQAs for each of the measuring system's functionalities using GMI transducer to locate non-ferromagnetic foreign bodies in patients.

The adaptation of the QbD approach to meet quality requirements from the earliest stages of the device development responds to a quality control strategy aimed at best practices for biomedical equipment development. The proposed method for adapting the QbD approach, applied in the development of the magnetic measuring system for locating non-ferromagnetic foreign bodies in 
patients, contributes to the quality assurance of this technology, in particular; and to the advancement of knowledge towards the implementation of the QbD in the development of biomedical technologies in general. Even though the Hybrid QbD-Fuzzy approach successfully dealt with the multiparameter's challenge for full implementation of $\mathrm{QbD}$ in a highly complex technology, further investigations considering biomedical technologies with characteristics diverse from the measurement system for diagnostic imaging studied, such as different operating mechanism or measured quantities, may provide complementary inputs. The Hybrid QbD-Fuzzy approach proposed for multiparametric DS configuration can be a powerful strategy for applying QbD procedure in medical devices' development, enabling the use of an additional and promising tool to promote reliable diagnosis and therapeutic interventions.

\section{Conflict of interests}

The authors declare that they have no conflict of interest.

The authors acknowledge the financial support provided by the Brazilian Agencies CNPq, FINEP, FAPERJ, and CAPES (Coordenação de Aperfeiçoamento de Pessoal de Nivel Superior) - Brazil - Financing Code 001.

\section{References}

1. D.M. Clarkson, Med. Eng. Phy. 41, 97-102 (2017)

2. T. Bayraka, F.Ö. Çopur, H. Pol. Tech. 6, 234-241 (2017)

3. F. Geremia, Micro. J. 136, 300-306 (2108)

4. MHRA, Managing medical devices: Guidance for healthcare and social services organisations, Medicines and Healthcare Regulatory Agency (MHRA), Department of Health, London, UK, 1-60 (2015). https://assets.publishing.service.gov.uk/ government/uploads/system/uploads/attachment_data/fi le/421028/Managing_medical_devices_-_Apr_2015.pdf (accessed January 10, 2020)

5. M.C. Ferreira, Int. J. Metrol. Qual. Eng. 2, 135-140 (2011)

6. E. Costa Monteiro, M.L. Lessa, Engev. 7, 51-60 (2005)

7. E. Costa Monteiro, Metrol. Inst. 6, 6-12 (2007)

8. E. Costa Monteiro, L.F. Leon, J. Phys.: Conf. Ser. 588, 1-6 (2015)

9. FDA (Food and Drug Administration). Pharmaceutical CGMPs for the 21st Century - A Risk-Based Approach: Final Report, 2004

10. FDA (Food and Drug Administration). Guidance for Industry PAT - A Framework for Innovative Pharmaceutical Development, Manufacturing, and Quality Assurance, 2004

11. ICH (International Conference on Harmonisation), Technical Requirements for Registration of Pharmaceuticals for Human Use: Pharmaceutical Development Q8-Approval of the Guideline by the Steering Committee under Step 4 and recommendation for adoption to the three ICH regulatory bodies, Guideline, 2005

12. ICH (International Conference on Harmonisation), Technical Requirements for Registration of Pharmaceuticals for Human Use: ICH Q9-Quality Risk Management, Guideline, 2005
13. ICH (International Conference on Harmonisation), Technical Requirements for Registration of Pharmaceuticals for Human Use: ICH Q10-Pharmaceutical Quality System, Guideline, 2008

14. ICH (International Conference on Harmonisation), Technical Requirements for Registration of Pharmaceuticals for Human Use: Pharmaceutical Development Q8 (R2), Guideline, 2009

15. A.M. Rivero, E. Costa Monteiro, D.S. Leite, F.S. Tannenbaum, M.A.G. Pinto, K.T.F. Leite, In: Proceedings of XXI IMEKO World Congress Measurement in Research and Industry, Prague, 1, 1295-1299 (2015)

16. D.R.T. Velázquez, D.R. Louzada, E. Costa Monteiro, L.G.S. Fortaleza, C.R.H. Barbosa, E.C. Silva, L.A.P. Gusmão, J. Phys.: Conf. Ser. 1044, 1-6 (2018)

17. N.P. Nadpara, R.V. Thumar, V.N. Kalola, P.B. Patel, Int. J. Pharm. Sci. Rev. Res. 17, 20-28 (2012)

18. H. Patel, S. Parmar, B. Patel, Int. J. Phar. Sci. Rev. Res. 21, 223-236 (2013)

19. D.R. Louzada, E. Costa Monteiro, L.G.S. Fortaleza, C.R.H. Barbosa, D.R.T. Velázquez, E.C. Silva, L.A.P. Gusmão, J. Phys.: Conf. Ser. 772, 1-6 (2016)

20. L. Zhang, S. Mao, Asian J. Pharm. Sci. 12, 1-8 (2017)

21. T. Bastogne, Nanomed: Nanotech. Biol. Med. 13, 2151-2157 (2017)

22. L.X. Yu, M. Kopcha, Int. J. Pharm. 528, 354-359 (2017)

23. J.J. Peterson, J. Biopharm. Stat. 18, 959-975 (2008)

24. J.J. Peterson, K. Lief, Stat. Biopharm. Res. 2, 249-259 (2010)

25. S. Mazumder, N. Pavurala, P. Manda, X. Xu, C.N. Cruz, Y. S.R. Krishnaiah, Int. J. Pharm. 527, 151-160 (2017)

26. H. Bhatiaa, E. Read, C. Agarabi, K. Brorson, S. Lute, S. Yoon, Int. J. Pharm. 512, 242-252 (2016)

27. H. Raina, S. Kaur, A.B. Jindal, J. Drug Del. Sci. Tech. 39, 180-191 (2017)

28. S. Dufreneix, C. Legrand, C. Di Bartolo, M. Bremaud, J. Mesgouez, T. Tiplica, D. Autret, Phys. Med., Eur. J. Med. Phys. 41, 26-32 (2017)

29. S. Dhat, S. Pund, C. Kokare, P. Sharma, B. Shrivastava, Eur. J. Pharm. Sci. 96, 273-283 (2017)

30. J. Djuris, Z. Djuric, Int. J. Pharm. 533, 346-356 (2017)

31. D. Hales, L. Vlase, S.A. Porav, A. Bodoki, L. BarbuTudoran, M. Achim, I. Tomuță, Eur. J. Pharm. Sci. 100, 249-261 (2017)

32. A.S. Rathore, G. Kapoor, F. Biop. Proc. 99, 231-243 (2016)

33. E. Costa Monteiro, C.R.H. Barbosa, E.A. Lima, P. Costa Ribeiro, P. Boechat, Phys. Med. Biol. 45, 2389-2402 (2000)

34. C.R.H. Barbosa, E. Costa Monteiro, E.A. Lima, S.F. Santos, E.G. Cavalcanti, P. Costa Ribeiro, Appl. Sup. IEEE Trans. 11, 677-680 (2001)

35. C.R.H. Barbosa, Rev. Sci. Inst. 75, 2098-2106 (2004)

36. L.G.S. Fortaleza, C.R.H. Barbosa, E. Costa Monteiro, E.C. Silva, L.A.P. Gusmão, Detecting Non-Magnetic Metallic Foreign Bodies by GMR Sensors through the Use of Eddy Currents, in: $8^{\circ}$ Congresso Brasileiro de Metrologia, 2015, pp. $1-4$

37. L.G.S. Fortaleza, E.C. Silva, C.R.H. Barbosa, L.A.P. Gusmão, E. Costa Monteiro, Elect. Lett. 55, 1808-1809 (2015)

38. L.G.S. Fortaleza, E. Costa Monteiro, C.R.H. Barbosa, E.C. Silva, L.A.P. Gusmão, J. Phys.: Conf. Ser. 1044, 012013 (2018) 
39. D.R. Louzada, E. Costa Monteiro, L.A.P. Gusmão, C.R.H Barbosa, Medição não-invasiva de ondas de pulso arterial utilizando transdutor de pressão MIG, in: IFMBE proceedings Springer Berlin Heidelberg, 2007, pp. 436-439

40. F.M.P. Cavalcanti, L.A.P. Gusmão, C.R.H. Barbosa, E. Costa Monteiro, L.A.P. Gonçalves, F.L.A. Machado, Meas. Sci. Technol. 19, 025801 (2008)

41. D. Martinez-Marquez, A. Mirnajafizadeh, C.P. Carty, R.A. Stewart, PLoS ONE, 13, 195-291 (2018)

42. D. Martinez-Marquez, A. Mirnajafizadeh, C.P. Carty, R.A. Stewart, Proc. Manuf. 30, 284-291 (2019)
43. J.E. Zimmerman, N.V. Frederick, Appl. Phys. Lett., 16-19 (1971)

44. L.A. Zadeh, Fuzzy Sets. Inform. Cont. 8, 338-353 (1965)

45. R. Tanscheit, Sistemas fuzzy. Inteligência computacional aplicada a administração, economia e engenharia em Matlab, 229-264 (2004)

46. F. Gomide, R. Gudwin, R. Tanscheit, In: Proc. 6th IFSA Congress-Tutorials, 1-38 (1995)

47. F.C. Gomide, R.R. Gudwin, SBA Cont. Autom. 4, 97-115 (1994)

48. ICNIRP, Health Phys. 99, 818-836 (2010)

Cite this article as: Daniel René Tasé Velázquez, Elisabeth Costa Monteiro, Daniel Ramos Louzada, Carlos Roberto Hall Barbosa, Multiparametric quality by design-fuzzy model applied in the development of a biomedical measuring system, Int. J. Metrol. Qual. Eng. 11, 12 (2020) 